A N N A L E S Annales de Bretagne et des Pays de l'Ouest

\title{
Marchand de La Houlière et la création de la fonderie de canons d'Indret (1775-1778)
}

Paul Naegel

\section{OpenEdition}

\section{Journals}

Édition électronique

URL : http://journals.openedition.org/abpo/217

DOI : 10.4000/abpo.217

ISBN : 978-2-7535-1512-3

ISSN : 2108-6443

Éditeur

Presses universitaires de Rennes

Édition imprimée

Date de publication : 31 décembre 2008

Pagination : $55-79$

ISBN : 978-2-7535-0808-8

ISSN : 0399-0826

\section{Référence électronique}

Paul Naegel, « Marchand de La Houlière et la création de la fonderie de canons d'Indret (1775-1778) »,

Annales de Bretagne et des Pays de l'Ouest [En ligne], 115-4 | 2008, mis en ligne le 31 décembre 2010, consulté le 01 mai 2019. URL : http://journals.openedition.org/abpo/217 ; DOI : 10.4000/abpo.217 


\title{
Marchand de La Houlière et la création de la fonderie de canons d'Indret (1775-1778)
}

\author{
Paul NAEGEL \\ Docteur en histoire des techniques \\ Université de Nantes - UFR des sciences et des techniques, \\ Centre François-Viète
}

Les faits évoqués dans cet article se sont déroulés pour l'essentiel entre 1775 et le premier semestre de 1778 , c'est-à-dire au début du règne de Louis XVI. Mais les événements qui nous intéressent ici ne sauraient être détachés d'autres, survenus durant la fin du règne de Louis XV, en particulier une guerre mettant aux prises plusieurs puissances, et pour ce qui nous concerne, la France et l'Angleterre. La défaite de la flotte française au large de Belle-lle, le 20 novembre 1759, marqua sans doute une date douloureuse pour les marins et officiers artilleurs de la Royale. Au cours d'un conflit qui avait commencé en 1756, cet engagement contribua à montrer la supériorité des canons anglais. Le 10 février 1763 prenait fin cette guerre dite de sept ans, et le traité de Paris était signé.

Dans la seconde moitié du xvIII siècle, il était devenu évident pour certains responsables de la marine de guerre française que celle-ci était inférieure à la marine anglaise. La forerie de canons de Ruelle, créée à partir de 1751 par René-Marc Marquis de Montalembert, n'était, semble-t-il, pas en état d'améliorer la situation. Et bien que l'on ait fait appel, en 1755, au fondeur d'origine suisse, Jean Maritz, inventeur d'une machine à forer les canons, la coulée de ceux-ci se faisait encore avec de la fonte de première fusion au charbon de bois. Ce procédé ne permettait pas d'égaler en qualité l'artillerie de la Royal Navy.

La possibilité de création d'une fonderie et forerie supplémentaire de canons, complètement nouvelle du point de vue des techniques utilisées, a donné lieu à une bataille, à la fois politique et technique, entre des groupes de pression rivaux auprès du pouvoir royal et de ses dignitaires en cour à Versailles. Ces difficultés résultaient en partie de la séparation des commandements, au plus haut niveau de l'État, entre l'armée de terre et 
la marine de guerre. La première avait ses fonderies de canons à Douai, Strasbourg et Metz, la seconde notamment à Ruelle, près d'Angoulême. Dans ces conditions, une innovation proposée par un membre, même éminent, de l'artillerie de terre, ne pouvait que susciter des doutes, et plus encore des réticences, chez les responsables de la Royale.

Car c'est bien une innovation qu'il fallait introduire dans la méthode de coulée des canons de marine. L'utilisation de fontes au charbon de bois en première fusion avait été abandonnée depuis des lustres par les Anglais, qui ajoutaient à celles-ci, produites avec du charbon de terre, des fontes ayant déjà servi. Ils obtenaient ainsi, en seconde fusion, de la fonte résistant mieux aux contraintes que subissaient les canons lors des tirs. Mais proposer une méthode étrangère, de surcroît anglaise, à un corps aussi sûr de lui que celui des officiers de la marine royale française ne pouvait qu'augmenter leur résistance. À ceci s'ajoutaient des considérations économiques, tant il pouvait paraître évident que l'emploi, pour partie, des vieilles fontes qui encombraient les arsenaux, devait se traduire par une réduction sensible du coût des nouveaux canons. La fonderie-forerie de canons d'Indret n'existait pas au moment où l'idée de créer une nouvelle fonderie-forerie est venue à un officier supérieur de l'armée de terre. Il serait donc, dans le cadre de cet article, anachronique de faire une étude comparative de ses performances avec celles obtenues à Ruelle.

Au cours de la période concernée par notre étude, la pénurie de bois commençait certes à se faire sentir en France, mais le pays était encore suffisamment pourvu en forêts pour que les techniques traditionnelles de la sidérurgie au charbon de bois puissent se maintenir, étant donné l'attachement des maîtres de forge à des habitudes ancestrales. Aussi n'est-il pas étonnant que l'argument de la pénurie de bois n'ait été utilisé que de manière très marginale par le promoteur d'Indret. Dans le même temps, les charbons de terre, inventoriés en France par des ingénieurs tels que Gabriel-Jean Jars, n'étaient pas appréciés pour la marche des hauts-fourneaux, car beaucoup d'entre eux donnaient des fontes cassantes, notamment à cause de la présence de phosphore. Il apparaît par ailleurs évident, à l'examen de la documentation, que la modernisation et la rationalisation de la production d'armes de guerre, que ce soit pour l'armée ou la marine, tant pour les armes légères que pour l'artillerie, étaient autour des années 1775 à l'ordre du jour, notamment sous l'impulsion de Gribeauval. Il eut d'ailleurs son mot à dire lorsque fut prise la décision de créer Indret.

Parmi les acteurs concernés par les enjeux complexes, relatifs à la future usine d'Indret, se trouve le brigadier-général Mathieu Henri Marchand de La Houlière, issu de l'armée de terre. Ce sont ses efforts, en tant qu'innovateur, que nous avons suivis quasiment au jour le jour. Il eut comme confident Charles-Claude de Langeron; le réformateur de l'artillerie de terre et de mer, Jean-Baptiste Vaquette de Gribeauval, a été son allié au moment décisif. Quant au fondeur et foreur de canons anglais qu'il arriva à faire accepter 
en tant que régisseur de la nouvelle fonderie et forerie d'Indret, encore à construire en 1777, c'était William Wilkinson ${ }^{1}$.

\section{Les protagonistes}

\section{Des Français recherchent des techniques métallurgiques en Angleterre}

Des voyages et des contacts établis par des Français éminents, généralement inspirés et soutenus par les autorités du moment, ont précédé le transfert effectif des techniques anglaises vers la France. Ce sont, au sens large, des techniques métallurgiques d'outre-Manche qui étaient recherchées, mais nous nous limiterons dans ce texte à celles en rapport avec l'établissement d'Indret.

Ainsi Gabriel-Jean Jars aurait séjourné en Angleterre, entre juillet 1764 et septembre 1765, pour enquêter sur les techniques métallurgiques ${ }^{2}$. Mais le rapport de son voyage avec l'armement de la marine royale française ne semble pas établi. Ensuite, en 1773, Le Camus de Limare, qui possédait une mine de charbon en Bourgogne, rencontrait John Wilkinson à l'occasion d'une visite faite à Boulton et Watt, pendant laquelle il offrit au premier d'agir pour lui en France ${ }^{3}$. On pourra relever également le voyage en Angleterre de Pierre-Augustin Caron de Beaumarchais, envoyé dans ce pays comme agent spécial par Louis XV, pour visiter des manufactures ${ }^{4}$. Nous ne savons pas s'il a rencontré John Wilkinson à cette occasion, mais l'auteur du Barbier de Séville a su se faire marchand de canons en 1776, pour le compte les Insurgents en Amérique du Nord. Rappelons également que c'est en 1774 que John Wilkinson a fait breveter sa machine à aléser les cylindres; il exploitait alors également des hauts fourneaux à New Willey, près de Broseley. Il apparaît donc que John Wilkinson n'était plus, à la suite de ces voyages, un inconnu pour certains Français intéressés par les techniques métallurgiques anglaises. Mais vers 1775, il s'agissait de couler et de forer des canons, en France, de manière plus performante et avec des résultats aux essais et à l'usage meilleurs que ceux qui se faisaient à Ruelle.

Marchand de La Houlière n'aura donc pas été le premier, en juillet-août 1775, à se rendre en Grande-Bretagne pour y rencontrer le fondeur dont la réputation avait déjà traversé la Manche, John Wilkinson. Mais cette

1. Une remarque s'impose d'abord : la technique anglaise qui consistait à couler des canons en fonte de seconde fusion ne se confond pas avec le procédé d'affinage, nommé puddlage, inventé par l'anglais Henry Cort en 1784. Cette autre technique était à l'origine des forges à l'anglaise, qui n'ont été introduites en France que pendant la Restauration, à partir de 1820 .

2. CotTe, Michel, De l'Espionnage industriel à la veille technologique, Besançon, Presses universitaires de Franche-Comté, 2005, 294 p.

3. Source : [http://www.adam-matthew-publications.co.uk/digital_guides/industrial_revolution/detailed\%20listing\%20-\%20part\%201.aspx].

4. Source : [http://www.broseley.org.uk/Docs/journal-00.PDF]. 
rencontre était décisive pour l'existence même du futur établissement d'Indret.

\section{Le brigadier-général Mathieu-Henri Marchand de La Houlière}

Il ne nous a pas été possible de dresser une généalogie satisfaisante de Mathieu-Henri Marchand ${ }^{5}$ de La Houlière, ni même de préciser sa date et son lieu de naissance. Parmi les maigres éléments trouvés, il y a ses probables prénoms, Mathieu-Henri, et son mariage (avant 1753) avec Marie-Agnès de Her. Au moins une fille serait née (vers 1753) de cette union, Marie-Françoise, qui décéda le 8 juin 1778 à Perpignan, après avoir épousé le 20 juin 1769 François-Antoine Le Moyne d'Aubermesnil ${ }^{6}$, à Salses-le-Château ${ }^{7}$.

Une allusion à notre personnage se trouve dans un ouvrage publié en 2003, sans nom d'auteur, à partir des mémoires, du journal et des lettres de Louis-Antoine de Bougainville, sous le titre, Écrits sur le Canada (p. 267). Les termes en sont les suivants : "M. de La Houlière, commandant de Salces, en Roussillon, ci-devant major de Lyonnais, avec brevet de colonel, est arrivé sur le Bizarre à Louisbourg pour commander les troupes françaises de la place. " Il s'agit là d'une référence faite par Louis-Antoine de Bougainville à la campagne de l'expédition d'Amérique en 1758, commencée le 23 juin de la même année. Cela porte à penser que Mathieu-Henri Marchand de La Houlière était déjà, avant 1758, commandant de la forteresse de Salces. Et il aura sans doute quitté le Canada avant 1769 pour reprendre son commandement en Roussillon, ce que suggère le mariage de sa fille en cette région.

Marchand de La Houlière serait, selon Jean Buvat ${ }^{8}$, le neveu de Voltaire, lequel « avait un cousin germain du nom de Marchand, qui était négociant, et eut deux fils, dont l'un, Marchand de La Houlière, était brigadier des armées du Roi en 1770; et l'autre, Marchand de Varenne, après avoir été maître d'hôtel du Roi, obtint l'emploi de fermier général ${ }^{9}$ ". Cette parenté semble confirmée par l'adresse, "chez M. de Varennes, fermier général, rue St. Louis ", donnée par La Houlière dans une lettre du 17 juin 1776 au marquis de Langeron ${ }^{10}$. Voltaire écrivit à La Houlière, depuis Ferney, le 22 octobre 1770, une lettre dans laquelle il le nomme « mon cher neveu à la mode de Bretagne, et non pas mon cousin ${ }^{11}$ ". Il répondait ainsi à la lettre

5. Le nom est quelquefois orthographié Marchant, voire Merchant. Très souvent, le nom est abrégé en de La Houlière ou encore de Lahoulière, voire en La Houlière. Dans cet article, nous avons opté de manière uniforme pour la graphie Marchand de La Houlière, abrégée éventuellement en La Houlière.

6. PAYENNEVILLI, Olivier, Base GENEANET.

7. Salses-le-Château est le nom actuel de Salces, dans les Pyrénées-Orientales (région Languedoc-Roussillon); une forteresse y est toujours visible.

8. Buvat, Jean, Journal de la régence (1715-1723), Tome II, Boston, Adamant MediaElibron Classics, 2001, 565 p.

9. Ibidem, p. 512 .

10. LANGERON, Charles Claude marquis de; de 1755 à 1790, il fut gouverneur des ports et château de Brest.

11. VolTAIRE, Euvres complètes, Tome LXI (1784), Lettre CCXVII ( 22 octobre 1770). 
que lui avait envoyée "son neveu " le 20 septembre 1770, et dans laquelle il se plaignait de "n'être point brigadier ». Et Voltaire de le rassurer, lui prouvant " qu'il l'est ", en citant une lettre du 14 octobre 1770 que lui-même avait reçue à ce propos, du duc de Choiseul. « Fi, que cela est mal de crier famine sur un tas de blé ", ironise l'écrivain. Dans une lettre du 5 août 1776 envoyée au marquis de Langeron, La Houlière lui demandera d'adresser sa réponse "sous la double enveloppe de M. de Meilhan ${ }^{12}$ ".

Il est clair, au vu des copies de lettres que nous avons analysées, que Marchand de La Houlière avait, au plus tard à partir de 1775, ses entrées à la cour, et cela auprès de personnages aussi considérables que le comte Charles Gravier de Vergennes, ministre des affaires étrangères (21 juillet 1774-13 février 1787). Il ne semble pas avoir eu non plus de difficultés particulières pour approcher Antoine-Raymond de Sartine, ministre de la marine. De même, il correspond avec le comte Claude-Louis de SaintGermain (15 avril 1707-15 janvier 1778), ministre et secrétaire d'État au département de la guerre (25 octobre 1775-23 septembre 1777).

Marchand de La Houlière exploitait aussi, dès 1773, à Alais un haut-fourneau ${ }^{13}$ qui marchait au coke et non au charbon de bois, ce que rapporte l'historien anglais William-Henry Chaloner ${ }^{14}$. Un tel mélange des genres entre des fonctions militaires et des activités industrielles n'était pas surprenant sous l'Ancien Régime, au cours duquel la noblesse, d'épée et de robe, a investi notamment dans la métallurgie, et plus généralement, a pris des intérêts dans diverses activités économiques. Une fois en retraite, comme il en a fait mention lui-même dans une lettre le 5 septembre 1776 au marquis de Langeron ${ }^{15}$, le brigadier général se rendit en Angleterre ${ }^{16}$ en juillet-août 1775, à la fois par intérêt personnel et, selon ses dires, en tant que chargé de mission par le ministre de la Royale, Antoine-Raymond de Sartine. Nous reviendrons sur ce voyage.

Marchand de La Houlière figurait encore, pour l'année 1789, dans l'État militaire de France, parmi les membres des gouvernements particuliers, en Roussillon, à Salces, en tant que lieutenant du roi, le comte de Rochambeau étant gouverneur. Aurait-il repris du service ou bien s'agissait-il d'un poste honorifique? Nous ne sommes pas en mesure de trancher. Mais la suite plaide pour une reprise de service au moment de la Révolution. Car sa fin semble avoir été tragique. Marchand de La Houlière était commandant en chef de l'armée des Pyrénées orientales, en garnison à la forteresse de

12. Il s'agit de Gabriel SÉNAC de MEILHAN, intendant de la guerre et des armées auprès du comte de Saint-Germain.

13. Le nom actuel de cette commune est Alès.

14. Chaloner, William Henry, Industry and Innovation - Selected Essays, London, Frank Cass and Company Limited, 1990, 318 p., p. 19.

15. Médiathèque de Nantes, MS 506 C4. Lettres de M. de La Houlière au comte de Langeron sur la fonte des canons. Lettre du 5 septembre 1776.

16. PRACA, Edwige, « 1775 : Le voyage métallurgique en Angleterre de Henri Marchand de La Houlière, commandant du fort de Salces ", Conférence de l'APHPO, Perpignan, Maison d'édition Trabucaire, 2004, 35 p. 
Salses, pendant la guerre de la Convention contre l'Espagne (1793-1795). Sa stratégie dans le conflit fut purement défensive. Le 20 avril 1793, la commune de Céret ${ }^{17}$ fut occupée par les Espagnols, et Perpignan menacée. Alors, " [... ] el viejo general La Houlière, destituido de su cargo, se suicidó por no poder soportar la vergüenza de la derrota y su destitución [... $]^{18}$ ".

Tels sont les modestes éléments de la biographie de ce brigadier-général que nous avons pu réunir.

\section{Le correspondant privilégié : Charles-Claude de Langeron}

Une grande partie de la correspondance expédiée par le brigadier-général a été adressée à Charles-Claude de Langeron. La notice relative au fonds Langeron ${ }^{19}$ aux Archives nationales indique : " Charles-Claude, comte puis marquis de Langeron (1720-1792), fut lieutenant-colonel du régiment de Condé en 1743, brigadier en 1748, maréchal de camp en 1758 et lieutenant général en 1762. De 1755 à 1790, il fut gouverneur des ports et châteaux de Brest. » Il s'agit bien là du correspondant privilégié de Marchand de La Houlière, lequel mentionne dans plusieurs lettres la présence de Langeron à Brest.

\section{Jean-Baptiste Vaquette de Gribeauval}

Jean-Baptiste Vaquette de Gribeauval (1715-1789), d'abord officier de l'artillerie royale, fut nommé inspecteur de l'artillerie en 1764. Il a participé dès lors à la modernisation du corps des mines et de l'artillerie. Après une période de disgrâce ayant débuté en 1774, rappelé en 1776 par le ministre de la guerre Claude Louis de Saint-Germain, il poursuivit son œuvre de rénovation et de rationalisation. Surtout connu pour le canon et le fusil qui portent son nom, il aura été, comme nous le verrons, partie prenante dans les tractations et la décision finale ayant conduit à la construction de la fonderie et forerie de canons d'Indret. Marchand de La Houlière l'avait rencontré au préalable à plusieurs reprises et avait réussi à le rallier à ses vues.

\section{Le rôle clé des frères John et William Wilkinson}

Il nous faut, avant d'en arriver à décrire les efforts de Mathieu-Henri de La Houlière, rappeler le rôle clé des frères Wilkinson dans la technique de fabrication des canons.

John Wilkinson (1728-1808), fils d'Isaac, avait en 1748 accumulé suffisamment de capital pour établir son propre haut-fourneau au coke près de Wolverhampton, après de nombreux essais infructueux, en joignant ses

17. Commune au sud de Perpignan, près de l'actuelle frontière franco-espagnole.

18. Source : site Internet [http://www.ingenierosdelrey.com/guerras/1793_francia/rosellon_93/04_20_ceret.htm].

19. Arch. nat., $118 \mathrm{AP}$, fonds Langeron. 
efforts à ceux de son père, déjà maître de forges et fondeur à Bersham. Le nom de Wilkinson semble avoir été connu en France autour de 1770, mais encore faut-il préciser s'il s'agissait de John ou de William, son frère cadet. Leurs destins furent très liés, bien que le véritable novateur fût incontestablement John. En quoi consistait son innovation? Dans la réalisation d'une machine à aléser les cylindres en fonte de sa fabrication, outil pour lequel il aura obtenu un brevet le 27 janvier 1774. Sans le savoir-faire de John Wilkinson en matière d'alésage, plus encore que son expérience de fondeur, la machine inventée par Watt aurait été difficile à réaliser de manière efficace. Seul John Wilkinson était à l'époque capable d'aléser au millimètre près des cylindres de grand diamètre. Et qui savait aléser correctement des cylindres pour des machines à vapeur, était également capable de bien aléser des canons.

L'établissement de la fonderie et forerie de canons d'Indret dépendait du bon vouloir des frères Wilkinson. Car il faut préciser que les meilleures affaires que John Wilkinson ait réalisées consistaient à couler " en plein ", puis à forer et aléser des canons, et finalement à les vendre avec d'excellents profits au plus offrant, dont bien entendu la Royal Navy. Quelques autres clients ${ }^{20}$, liés aux Insurgents pendant la guerre d'indépendance des futurs États Unis, lui ont valu des accusations d'intelligence avec l'ennemi, mais elles n'eurent pas de suite judiciaire.

\section{Voyages en Angleterre}

Dans la vie de Marchand de La Houlière, deux choses intéressent particulièrement la création d'Indret : son voyage en Angleterre (juillet-août 1775) pour approcher les Wilkinson et tenter de les intéresser au projet de la construction d'une fonderie et forerie de canons dans ce pays; ses interventions indirectes et directes, entre 1775 et 1778 , auprès des plus hauts responsables gouvernementaux, à la cour, pour obtenir la décision d'établir la fonderie et forerie d'Indret avec l'aide de William Wilkinson.

\section{Les voyages du brigadier-général Marchand de La Houlière (1773-1775)}

Tout semble avoir commencé par un voyage en Angleterre au cours de l'année 1773; dans un mémoire en date du 17 juin 1776 destiné au comte de Saint-Germain, La Houlière a écrit " [qu'il] a fait depuis trois ans beaucoup de recherches, de dépenses et de voyages [...] pour connaître tous les avantages que les Anglois [sic] tirent du charbon de terre dans leurs fourneaux et forges, et leur manière de l'employer avec succès, découverte qui devient aujourd'hui très intéressante par la rareté et cherté du bois [...] ".

20. Une société écran créée, sous le nom de "Roderique Hortalez et Compagnie " par Beaumarchais, pour exporter en secret des canons en 1776 aux Insurgents d'Amérique du Nord, semble avoir fait partie des clients de John Wilkinson. 
Mais ce voyage, s'il a eu lieu, n'est connu que par la citation ci-dessus. Il en est autrement du voyage de 1775, car dans le même mémoire, de La Houlière poursuit ainsi : “ [...] Au retour d'un voyage qu'il [La Houlière] a fait dans cette vue [utiliser du charbon de terre à la place du bois devenu rare] l'année dernière [donc en 1775] en Angleterre par ordre du Ministère, il a rendu compte à Monsieur de Sartine de la façon dont les Anglois [sic] refondent dans des fourneaux de réverbère où on ne consomme que du charbon de terre tous les canons de leur marine $[\ldots]^{21}$."

La Houlière précisait que c'est " par ordre du Ministère " qu'il s'était rendu en Angleterre. Mais de quel ministère? Sans doute celui de la guerre; son titulaire était alors le comte de Saint-Germain, dont il relevait comme faisant partie de l'infanterie, et non du ministère de la marine. L'information donnée au ministre de Sartine, en charge de la marine, était donc probablement due simplement à une pratique, sans doute courante, au sein du conseil du roi. Mais lisons la suite du mémoire: " [...] La fonte, au lieu d'être coulée comme en France en sortant du haut-fourneau, ainsi qu'on le pratiquait en Angleterre il y a vingt ans, est mise une seconde fois en fusion dans des fourneaux à air et réverbère où recuite et plus épurée elle se condense plus et acquiert beaucoup plus de cohérence et de résistance $[\ldots]^{22}$. " Beaucoup est dit, du point de vue technique, dans cette citation.

En ce qui concerne les sources anglaises, on trouve chez Chaloner le texte suivant :

" [...] In about 1770, John Wilkinson (1728-1808) and his brother William [?1744-1808] joint owners of an important ironwork at Bersham, near Wrexham in North Wales had succeeded in replacing charcoal by coke in their blast furnace. However in July-August 1775, Marchant de La Houlière, a brigadier in the infantry of Louis XVI, in the course of a "metallically journey" trough the main regions of the English iron industry, had studied for himself the processes of coke smelting, with which he himself had experimented, no without success, in 1773. He had secured a subsidy for this journey from the Estate of Languedoc and on his return he prepared an official report which is preserved in the Archives Nationales in Paris.

Provided with a letter of introduction from Messrs. Boulton \& Fothergill of Soho, near Birmingham, and accompanied par the Irish-man Mac Dermott, chaplain of the French ambassador in London, he met John Wilkinson at his second ironworks near Broseley in August 1775 [M de La Houlière to Boulton and Fothergill, August 31, 1775, originally preserved in the Birmingham Assay Office, but now transferred to the Birmingham Reference Library].

Wilkinson had a long conversation with him and gave him a letter of introduction to his brother William at Bersham.

Later in his report, de La Houlière described the two interviews in the third person and without identifying his hosts $[\ldots]^{23}$."

21. Médiathèque de Nantes, MS 505 C3. Mémoires sur l'artillerie de Marine, sur la fonderie d'Indret et la refonte des vieux fers coulés 1776-1778. Mémoire du 17 juin 1776.

22. Médiathèque de Nantes, MS 505 C3. Mémoire sur la refonte des vieux fers coulés, 17 juin 1776.

23. CHALONER, William Henry, Industry and Innovation..., op. cit., p. 19. 
L'examen de ce texte indique que le Français n'avait pas contacté directement John Wilkinson lors de son déplacement. C'est en rendant visite à MM. Boulton et Fothergill à Soho ${ }^{24}$, comme le montre son courrier du 31 août 1775 adressé à ces messieurs ${ }^{25}$, que La Houlière obtint d'eux une lettre de recommandation pour visiter l'une des usines de John Wilkinson, à Broseley. Il fut alors reçu pendant deux semaines par celui-ci; il était accompagné, comme traducteur, par l'irlandais MacDermott, chapelain de l'ambassadeur de France à Londres, Adrien-Louis de Bonnières, comte (et plus tard duc) de Guines. La Houlière eut une longue conversation avec John Wilkinson, qui lui donna une lettre d'introduction auprès de son frère cadet William, installé à Bersham ${ }^{26}$, et encore célibataire. Car John Wilkinson n'était pas disposé à venir en France et préférait veiller à ses affaires en Angleterre. Il ne voulait pas non plus traiter avec un gouvernement dont il jugeait l'existence précaire, et il aurait préféré une entreprise privée. Il orienta donc son visiteur sur son frère cadet, William. La Houlière fut plus convaincant avec celui-ci, et sut l'intéresser à l'idée de construire et diriger en France une fonderie et forerie de canons pour la marine royale. Il lui promit non seulement un contrat pluriannuel avec une forte rémunération, le remboursement de ses frais, mais aussi la disposition d'une maison. À la suite de ce voyage, La Houlière a rendu un rapport officiel au gouvernement français, adressé à Monsieur de Sartine ${ }^{27}$; ce texte est conservé aux Archives nationales ${ }^{28}$. Des extraits en ont été traduits et publiés par Chaloner ${ }^{29}$.

Il semble bien que La Houlière se soit montré suffisamment persuasif, puisque William Wilkinson, conduit par le comte de Guines, accepta de traverser la Manche pour une première visite en France qui commença peu avant le 3 décembre $1775^{30}$. Un contrat en bonne et due forme entre le ministre Sartine et Wilkinson n'est intervenu que plus tard, après que des essais comparatifs eussent été faits entre les méthodes et les matières à utiliser, tant françaises qu'anglaises, pour la coulée de canons.

En attendant l'arrivée des canons dont il avait demandé l'expédition en France, lors de ce premier voyage, William Wilkinson visita, en compagnie du brigadier-général, plusieurs forges du royaume, où les mines de fer et de charbon étaient voisines. Une telle proximité était évidemment recherchée pour éviter des frais de transport. Il rejoignit également Alais, pour examiner le fourneau qu'y avait établi La Houlière ${ }^{31}$. La région en aval de Nantes, et notamment l'île d'Indret, semblent également avoir été visitées.

24. Ces deux associés avaient développé des machines à fabriquer des pièces par emboutissage, dont des monnaies.

25. ChALONER, William Henry, Industry and Innovation..., op. cit., p. 19.

26. Ibidem.

27. Ibid., p. 19-20.

28. Arch. nat., F12/1300.

29. CHALONER, William Henry, Industry and Innovation ..., op. cit., p. 19-20.

30. Ibidem, p. 20.

31. Ibid. 
Il apparaît également que la demande de livraison de canons de sa fabrication, faite par Wilkinson, ait été rapidement traitée. En effet, le comte de Givry écrivit le 23 décembre 1775 au vicomte Weymouth ${ }^{32}$, secrétaire d'État aux Affaires du Sud (1775-1779), lui demandant l'autorisation nécessaire pour l'expédition vers Rouen de 8 canons de différents calibres ${ }^{33}$. Il est possible, selon Chaloner, que ces canons aient finalement été débarqués à Brest au lieu de Rouen ${ }^{34}$, ce qui est confirmé par une note reçue du Bureau de la marine par La Houlière. C'est ensuite que les choses sont devenues plus difficiles et ont nécessité beaucoup d'activité de la part du brigadier général.

\section{Les interventions de Marchand de La Houlière auprès de la cour (1777-1778)}

Rien ne semble s'être passé, depuis la remise du rapport de voyage en Angleterre par La Houlière au ministère de la marine, si ce n'est la demande faite aux autorités britanniques pour l'acheminement en France de 8 canons fabriqués par Wilkinson. Le brigadier général a reçu alors du bureau de la Marine une note ainsi rédigée :

" Il a été remis à Brest le 16 juin dernier [1776] par le Sloup (sic) anglais la Catherine 8 canons de fer, 320 boulets et 4 saumons de fer de la même matière que les canons. Quant aux matériaux pour la construction d'un fourneau à réverbère, il y a lieu de croire qu'ils ont été remis à Nantes et transportés à la forge du S. Frerot près de cette ville, où le Ministre marque qu'il doit être coulé, au moyen du fourneau à réverbère qui y est établi, 4 canons de 4 dont l'épreuve sera faite avec 4 autres canons de même calibre fabriqués à la forge de Ruelle ou à celle du S. La Mothe. "

Dès le lendemain, La Houlière adressa à son correspondant, le marquis de Langeron, encore à Paris, la lettre suivante :

" J'ai l'honneur de vous adresser un Mémoire sur les canons qui doivent être éprouvés à Brest dont M. le Cte de St. Germain vous a parlé hier. Je n'ai aucun intérêt à la chose et mon seul motif est de procurer à l'Etat de meilleurs canons de fer que ceux qui ont été fondus jusqu'ici. Il serait fâcheux que faute d'un examen judicieux on rejette une Découverte qui mettrait en sûreté la vie des canonniers et mettrait fin aux accidents arrivés plusieurs fois sur nos vaisseaux. Il est heureux que votre Commandement vous conduise à Brest dans cette circonstance et que vous soyez à portée d'assister à ces épreuves avec les dispositions d'un juge sans prévention. Oserais-je vous supplier Monsieur de vouloir bien me faire la grâce de m'informer de ce que vous en pensez. J'ai l'honneur d'être $[\ldots]^{35}$."

32. Il s'agit de Thomas Thynne (13 septembre 1734-1796), homme politique britannique connu sous le nom de Lord Weymouth, vicomte Weymouth.

33. CHALONER, William Henry, Industry and Innovation..., op. cit., p. 30, note 6.

34. Ibidem.

35. Médiathèque de Nantes, MS 506 C4. Lettres de M. de La Houlière au comte de Langeron sur la fonte des canons. Lettre du 17 juin 1776. 
Le titre du mémoire que Marchand de La Houlière a envoyé en copie au marquis de Langeron est Mémoire sur la refonte des vieux fers coulés ${ }^{36}$. Il était complété par un supplément non daté, envoyé sans doute quelques jours plus tard au ministre de la guerre. Le texte de cette note, dont nous avons déjà utilisé de courts passages, est capital, ce qui nous conduit à en citer une plus grande partie commençant par son début :

" Le S[ieu]r Marchant de La Houlière, Brigadier des armées du Roy, son Lieutenant au Gouvernement de Salces, Roussillon, a fait depuis trois ans beaucoup de recherches, de dépenses et de voyages pour connaître tous les avantages que les Anglois [sic] tirent du charbon de terre dans leurs fourneaux et forges, et leur manière de l'employer avec succès, découverte qui devient aujourd'hui très intéressante par la rareté et cherté du bois.

Au retour d'un voyage qu'il a fait dans cette vue l'année dernière en Angleterre par ordre du Ministère, il a rendu compte à M. de Sartine de la façon dont les Anglois refondent dans des fourneaux de réverbère, où on ne consomme que du charbon de terre, tous les canons de leur marine.

La fonte, au lieu d'être coulée comme en France en sortant du haut-fourneau, ainsi qu'on le pratiquait en Angleterre il y a vingt ans, est mise une seconde fois en fusion dans des fourneaux à air et réverbère où recuite et plus épurée, elle se condense plus et acquiert beaucoup plus de cohérence et de résistance, ce qui fait que ces canons supportent l'effort de la poudre sans crever et évitent à ceux qui les servent les accidents malheureusement trop ordinaires aux nôtres. Tous les marins sont convaincus que ceux de la Fonderie d'Angleterre sont infiniment supérieurs, ce qui n'est dû qu'à la seconde fusion.

Appuyé par M. Le Comte de Vergennes et par M. Le Duc de Guines, le Sr. De La Houlière a attiré cet hiver un anglois très expert en cet art avec qui il a fait une tournée dans les forges de plusieurs Provinces du Royaume. M. de Sartine est convenu avec lui d'un traitement considérable pour venir établir en France une fonderie pour les canons de fer, et il est attendu incessamment avec des canons dont M. Le Comte de Vergennes a fait demander la sortie au Ministère Britannique.

Le Sr. De La Houlière encore plus directement attaché au service de terre ne serait pas satisfait si le soin qu'il a pris ne devenait pas utile d'un autre coté au département de la guerre qu'il a eu principalement en vue dans ses recherches.

Les fourneaux de réverbère dont on fait usage pour fondre les canons servent aussi journellement en Angleterre à refondre tous les vieux fers coulés, canons, boulets, bombes, marteaux défectueux, tuyaux, plaques, marmites cassées; tout y est remis à neuf et cette expérience peut être d'une grande épargne et d'une grande utilité pour le service de l'artillerie de Terre.

Sans entrer dans les plans d'économie de M. Le Comte de Saint Germain, le Sr. De La Houlière a cherché à savoir les besoins actuels de l'artillerie en fonte moulée. Il résulte de ces informations qu'il manque dans les Arsenaux du Royaume une prodigieuse quantité de bombes, obus et boulets de certains calibres indispensablement nécessaires, tandis qu'il y en a d'autres irrégulières ou moins en usage aujourd'hui dont on est surchargé.

36. Médiathèque de Nantes, MS 505 C3. Mémoires sur l'artillerie de Marine, sur la fonderie d'Indret et la refonte des vieux fers coulés 1776-1778. Mémoire du 17 juin 1776. 
Il existe de ces munitions irrégulières ou excédantes ou superflues 192.420 quintaux qui étant de trop sont par conséquent inutiles.

Si pour en débarrasser les Arsenaux et faire place aux munitions dont on manque, celles qui sont en trop seraient vendues [...] elles ne produiraient par conséquent au plus que 374.840 [livres].

$\mathrm{Au}$ lieu de vendre ces bombes et boulets ils pourraient être refondus dans les arsenaux principaux et être remis à neuf, soit en mortiers et affûts de fer, ou en munitions de calibre qui seraient ordonnés et qu'on jugerait les plus essentiels [...]."

Suit alors dans ce mémoire un développement assez confus, tant du point de vue technique que sur le bénéfice "économisé " pour le roi, dont le sous-titre est " Manière de procéder à ces fontes. " L'argumentation du brigadier-général repose sur deux idées essentielles : les Anglais obtiennent de meilleurs canons en utilisant des fontes de seconde fusion; c'est l'aspect technique; il y a dans les arsenaux français, notamment à Douai, Metz et Strasbourg, des centaines de milliers de quintaux de vieilles fontes, qu'il serait plus économique de réutiliser plutôt que de les vendre à vil prix, tout en utilisant pour la coulée des canons seulement des fontes neuves et chères, issues directement des hauts-fourneaux marchant au charbon de bois.

Le 24 juillet 1776, le marquis de Langeron informa La Houlière que les canons anglais, arrivés à Brest le 16 juin 1776, y étaient encore, ce qui donna lieu immédiatement à un mémoire que le brigadier-général remit au ministre de Sartine. Mais celui-ci lui demanda de le "réformer ", pour en expurger tout ce qui avait trait " à la personne " que La Houlière soupçonnait de provoquer des retards dans les opérations prévues pour les essais comparatifs. Cette personne était André Fougeroux de Secval ${ }^{37}$, un officier d'artillerie de la Marine qui avait eu la haute main sur la fonderie de canons de Ruelle.

La Houlière essayait de " garder la main " sur ce qui devait se faire pendant un temps loin de ses yeux, en profitant de la connaissance qu'il avait du marquis de Langeron nommé à Brest. Car il semble bien qu'à la suite de son nouveau rapport, il n'avait plus, au moins pour un temps, l'oreille et les faveurs du ministre Sartine. On peut aussi penser que celui-ci voulait privilégier son département, et peut-être Monsieur de Secval dans cette affaire, et tenir La Houlière, et plus généralement l'armée de terre, aussi loin que possible des développements à venir.

Le malentendu est dévoilé dans tous ses détails dans une nouvelle lettre que La Houlière adressa, depuis Issy près de Paris ${ }^{38}$, le 5 août 1776 , au marquis de Langeron, toujours à Brest. Il nous faut, pour la compréhension des difficultés que le brigadier général en retraite a eues avec les officiers d'artillerie de la Marine, en citer un large extrait.

37. Secval est quelquefois orthographié Serval dans certains documents.

38. De nos jours Issy-les-Moulineaux. 
"En vous adressant le Mémoire que j'avais eu l'honneur de vous lire, je comptais vous donner avis du départ des ordres de M. le comte de St. Germain et de ceux M. de Sartines. Je savais qu'on avait ordonné de faire partir de Brest quatre canons anglois pour en faire couler huit pièces de mêmes proportions en Angoumois. Lesquelles devaient être éprouvées par comparaison moitié à Brest, moitié à Rochefort. J'imaginais que ses ordres ayant été donnés le 30 juin ces huit pièces seraient coulées et envoyées à leur destination dans le courant de juillet. Et dans cette persuasion j'avais écrit à M. le [?] pour lui rappeler les ordres qui vous étaient nécessaires et à $\mathrm{M}$. Dangenoust ${ }^{39}$ pour se rendre sur les lieux. Il m'a marqué par sa réponse :

"Qu'ayant conféré sur cet objet avec M. Potier, premier commis de la Marine, il lui avait dit que cette fonte exigera du temps et on ne présume pas que les pièces françaises puissent être exécutées cette année. Qu'au surplus ce premier commis est convenu que M. le Cte de St. Germain serait informé des opérations que le département de la marine ferait à cet égard afin que ce Ministre prévienne M. le Marquis de Langeron ainsi que M. Dangenoust du temps où les épreuves pourront être faites."

Apprenant d'un autre côté par la lettre dont vous m'avez honoré le 24 du passé que ces canons étaient encore à Brest, j'ai remis à M. de Sartines un Mémoire dont je joins ici copie. Ainsi je n'ai pas à me reprocher de ne pas lui avoir dit les causes du retardement et les suites qu'il aurait. Il m'a "répondu qu'il sentait sur qui tombaient mes soupçons; qu'il ne lui convenait pas d'agir sans lui en cette circonstance; de supprimer de mon Mémoire ce qui regardait cette personne à qui il devait le renvoyer, et qu'il lui ordonnerait d'exécuter tout ce que je demandais avec la plus grande promptitude".

J'ai donc réformé mon mémoire mais vous prévoyez comme moi M. le Marquis qu'il y aura des lenteurs. C'est ainsi qu'on mine, qu'on dégoûte, qu'on rebute ceux qui proposent des choses utiles. Vous exigez que je vous parle comme à un ancien camarade. Je le fais sans restriction pour que vous soyez instruit; mais si vous écrivez à M. de Sartines à ce sujet ne paraissez pas être informé par moi. Votre lettre tomberait sans doute entre les mains de celui qui inspire les lenteurs. Contentez vous de lui représenter qu'on pourrait toujours éprouver à Brest les quatre canons Anglais avec les quatre Pièces françaises qu'on jugera les meilleures et qu'on peut dès le moment savoir à quoi s'en tenir quant à la résistance de la Poudre et la force de la fonte; et qu'on comparera ensuite les portées avec les pièces qui seraient fondues en Angoumois, qui ne pourront manquer d'être les mêmes. L'Ame des pièces et leurs longueurs et proportions ne diffèrent pas. C'est avec peine que je vois que ces lenteurs peuvent nous faire perdre l'occasion d'avoir un homme aussi expert que M. Wilkinson pour introduire dans notre service une méthode aussi utile. Que ces délais le feront arriver dans une saison où on ne pourra plus bâtir, et que c'est différer d'au moins un An cet établissement. Vous ne manquerez pas de faire les mêmes réflexions que moi. Vous avez comme moi prévu ces difficultés parce que nous vivons à peu près depuis le même temps $[\ldots]^{40}$."

39. Sans doute Louis-Claude d'Angenoust, chef de brigade au corps royal de l'Artillerie.

40. Médiathèque de Nantes, MS $506 \mathrm{C} 4$. Lettres de M. de La Houlière au comte de Langeron sur la fonte des canons. Lettre du 5 août 1776. 
Des épreuves de quatre canons anglais ont été faites à Brest le 24 août 1776, ce dont le marquis de Langeron a rendu compte à La Houlière par lettre du 30 août 1776 . On perçoit dans la réponse de ce dernier ${ }^{41}$, expédiée le 5 septembre 1776, toute son amertume; il donne quand même encore des explications techniques à son " camarade ". Mais il apparaît également dans ce courrier que La Houlière espérait que le Ministère lui accorderait une " gratification " qu'il évalue à 40000 livres, pour sa " découverte ". Il est cependant conscient que « les coffres du Roy sont trop obérés ». Il en vient finalement à se contenter du « plaisir d'avoir employé le loisir de sa retraite d'une façon à être encore utile au Roy ".

Les choses semblent pourtant s'être arrangées pour lui, car le 11 septembre 1776 il pouvait écrire au marquis de Langeron :

" J'allai samedi [ 7 septembre 1776] à Versailles comme j'avais eu l'honneur de vous en prévenir. M. le Cte de St. Germain me fit lire la suite des expériences faites les 28 et 29 [août 1776] dont il me parut très satisfait, ainsi que M. de Sartines, et il a été arrêté et convenu d'écrire incessamment à notre Anglais de venir avec les Patrons, Moules [...] qui lui avaient été demandés précédemment.

M. Pothiers avait raison de dire que les canons qu'on voulait faire couler à Ruelle ne pouvaient être faits cette année, les fourneaux de Ruelle et de la Mothe étant tombés; on y travaille, mais on ne pourra pas y fondre avant le mois de juin prochain, car il n'y a pas de charbon [...]; on a pris le parti de faire couler quatre pièces de 4 avec des gueuses provenant de Ruelle et de vieux canons du même endroit dans un fourneau de réverbère qui est à Nantes et qui travaille depuis quatre ans sous la conduite d'un nommé Frerot, ouvrier très expert. Notre Anglais n'arrivera guère qu'au commencement d'octobre, et s'il se présentait quelques obstacles à son voyage, nous opérerions sans lui, M. Dangenoust, M. de Secval chargé des fontes à Ruelle, et moi. M. de Sartines désire d'avoir encore cette expérience avant de faire la dépense d'une fonderie en grand.

M. de St. Germain est, ainsi que M. de Gribeauval, très empressé de mettre cette manière de fondre en usage, et je pense que cet empressement vient du compte que vous avez bien voulu rendre, et de l'état de nos batteries des côtes $[\ldots]^{42}$."

De La Houlière semblait donc être à nouveau optimiste puisqu'il écrivit sur un ton moins plaintif, le 18 septembre 1776, une lettre au marquis de Langeron, qu'il remercia avec hauteur d'une proposition que ce dernier lui avait faite le 13 du même mois :

" J'ai reçu hier la lettre que vous m'avez fait l'honneur de m'écrire le 13 [septembre 1776] et la note sur les forges que possède aux environs de la Charente un Capitaine d'Infanterie de votre connaissance.

Je n'oublierai certainement pas, Monsieur, cette recommandation de votre part, s'il est question d'un établissement qui puisse consommer plus

41. Médiathèque de Nantes, MS 506 C4. Lettres de M. de La Houlière au comte de Langeron sur la fonte des canons. Lettre du 5 septembre 1776

42. Médiathèque de Nantes, MS $506 \mathrm{C} 4$. Lettres de M. de La Houlière au comte de Langeron sur la fonte des canons. Lettre du 11 septembre 1776. 


\begin{abstract}
de gueuses que les fourneaux de la Mothe et de Ruelle n'en pourront fournir, mais je n'y vois pas d'apparence, attendu que par la Méthode proposée, on en emploiera peu, puisqu'on refondra les vieux canons qui entreront pour beaucoup dans la composition des nouveaux; c'est un des avantages qui résultent de ce procédé de refondre la vieille vaisselle sur le lieu même. Les ordres nécessaires pour faire à Nantes quelques canons pareils à ceux d'Angleterre ont été expédiés par M. de Sartines, et nous opérerons, j'espère, avec M. Dangenoust et M. de Secval dans le courant d' $8^{\text {bre }}$ [octobre]. Lorsqu'il y aura quelque chose de nouveau à cet égard, j'aurai soin de vous en rendre compte.

J'ai l'honneur $[\ldots]^{43}$."
\end{abstract}

L'espoir que manifestait de La Houlière de voir couler des canons à Nantes dès octobre 1776 était, nous le verrons, prématuré. Mais il semble bien que cette fois les vues du brigadier-général avaient été prises en considération, et qu'il allait pouvoir intervenir personnellement dans les essais comparatifs qu'il avait préconisés.

\title{
Les opérations techniques suscitées par le brigadier-général
}

Des coulées de canons étaient envisagées à Nantes, car le brigadiergénéral était préoccupé par l'inventaire des ressources métallurgiques disponibles dans les environs de cette ville. Il avait déjà indiqué dans une correspondance que par la Loire, il serait aisé de distribuer les matériels refondus, munitions et canons, vers les ports.

\section{À la recherche de ressources locales}

Dans un mémoire ${ }^{44}$ malheureusement ni daté ni signé, mais d'une écriture que l'on peut attribuer à La Houlière, ou à son secrétaire, le premier a décrit par le menu ce que savait faire un fondeur des environs de Nantes, le sieur Frérot, " qui y a un fourneau a air ou à réverbère ${ }^{45}$ ". Ce fourneau, qui devait servir par la suite pour des essais de coulée, était situé près de l'actuelle commune de Saint-Sébastien-sur-Loire. Le même Frérot exploitait également une forge à Clisson, dans laquelle il transformait les crasses résiduelles de son four en fer de bonne qualité.

Le document, intitulé "Mémoire sur une fonderie de canons de fer à établir à Nantes ", montre que l'on pouvait se procurer, comme le faisait le sieur Frérot, les matériaux, notamment des briques pour l'intérieur du fourneau, nécessaires à la construction d'un four à réverbère à des prix avantageux, même en les faisant venir de Nevers, en même temps que du charbon de terre ou des gueuses de fonte. Dans ce mémoire, La Houlière

43. Médiathèque de Nantes, MS 506 C4. Lettres de M. de La Houlière au comte de Langeron sur la fonte des canons. Lettre du 18 septembre 1776.

44. Médiathèque de Nantes, MS 505 C3. Mémoire sur une fonderie de canons de fer à établir à Nantes, sans date, sans signature.

45. Ibidem. 
écrivait : "On trouve très à portée de Nantes de bonne terre à mouler, de bon sable pour le sol des fourneaux ${ }^{46}$."

Pour ce qui est des gueuses de fonte, elles arrivaient de Bourgogne par la Loire. Mais on pouvait en trouver également, selon La Houlière, à « Ruelle et autres fourneaux de l'Angoumois, par la Charente et La Rochelle, et elles seraient à aussi bon marché, voire peut-être à meilleur prix ". Il montrait ensuite que si on refondait de vieux canons mélangés à de la fonte neuve, le quintal de fonte reviendrait seulement à un peu plus de 7 livres, là où l'utilisation unique de fontes neuves faisait monter ce prix à plus de 9 livres. Le raisonnement est poursuivi en imaginant que quatre fourneaux de la taille de celui du sieur Frérot pourraient, en marchant ensemble, livrer de quoi couler un gros canon par jour, voire deux si on utilisait huit fourneaux. C'est la première solution qui a été retenue plus tard à Indret.

\section{Des essais comparatifs de coulées de canons (décembre 1776)}

Les essais comparatifs qu'avait proposés La Houlière allaient pouvoir se poursuivre selon ses vues à Nantes, plutôt qu'à Ruelle, pour ce qui est de la coulée de canons. Mais à nouveau, des lenteurs se sont manifestées, dont se plaignit de La Houlière au marquis de Langeron le 2 octobre 1776 :

" Il ne tiendra pas à moi que le temps perdu ne soit bientôt réparé et avant notre départ, je compte en proposer les moyens, qu'on acceptera certainement, si les bruits dont on murmure dans le public ont quelques fondements.

Vous serait-il possible, Monsieur, de m'envoyer un Plan avec échelle des Aubusiers-Canons que vous désireriez être fondus, et une note des proportions et épaisseurs convenables. Je jugerai par leur poids s'il sera possible d'en couler quelqu'un pour essai dans le fourneau à réverbère du Sieur Frérot dont je connais la capacité.

Je vous rends mille grâces, Monsieur, de m'avoir fait passer l'état de ce qui est arrivé d'Angl.[eterre] par le Sloup la Catherine; il m'avait déjà été fourni des Bureaux de la Marine.

J'ai l'honneur d'être [...]"

Il y a un ajout en fin de lettre :

« J'attendais de vous répondre pour vous donner des nouvelles de notre Anglais, et nous n'en avons pas encore; s'il avait changé d'avis, nous nous en passerions ${ }^{47}$."

Voilà donc La Houlière ragaillardi, et prêt à se passer de Wilkinson! Et le lendemain, il écrivait de nouveau au marquis de Langeron à Brest, en lui tenant quasiment la main pour écrire au comte de Saint Germain :

"J'ai reçu en son temps la lettre que vous m'avez fait l'honneur de m'écrire le $207^{\text {bre }}$ [septembre 1776]. Vous êtes trop juste pour imputer le

46. Ibid.

47. Médiathèque de Nantes, MS 506 C4. Lettres de M. de La Houlière au comte de Langeron sur la fonte des canons. Lettre du 2 octobre 1776. 
retard de ma réponse à autre cause que l'indécision dans laquelle on m'a laissé, jusqu'à hier au soir seulement, que le départ de M. Dangenoust, celui de M. de Secval et le mien pour Nantes a été décidé. Nous nous y rendrons le 25 du courant [ 25 novembre 1776] pour éprouver le degré de perfection que nos vieilles fontes acquerront étant de nouveau fondues et mélangées, en proportions dues, dans des fourneaux à réverbère.

Nous y fondrons quelques pièces de 4 conformément au Modèle Anglais qui y est; et s'il est praticable d'exécuter dans ce four à réverbère l'Obusier-Canon que vous pensez pouvoir être aussi utile au service, il en sera certainement fondu un pour prouver la possibilité d'en couler à Nantes en plus grand nombre suivant vos désirs, et prouver qu'on peut en faire davantage.

Au reste vous auriez le temps d'écrire à $M$. le Cte de St. Germain qu'étant instruit que nous sommes à Nantes pour faire des épreuves "conformément aux ordres qui ont été donnés par luy, de concert avec M. de Sartines, il serait utile au service d'y faire couler, si le fourneau à réverbère peut le permettre, un Obusier-Canon suivant le Plan que vous m'avez envoyé, et de vous adresser les ordres à l'effet de le couler, si nous trouvons les commodités convenables dans cette petite fonderie".

Je ne pense pas, M. le Marquis, que vous passiez l'hiver en Bretagne; je me flatte bien de pouvoir vous faire ma cour à Paris et de raisonner fontes et Poudres à canon.

Je vous rendrai compte de mon arrivée à Brest, et des facilités ou obstacles qui se rencontreront à couler l'Obusier-Canon. Vous êtes le Patron par la seule raison de l'avantage qui en peut résulter.

J'ai l'honneur d'être $[\ldots]^{48}$."

Mais il se trouve que le marquis était le 4 octobre à Paris, ou plus précisément à Fontainebleau, où la cour s'était transportée avec les ministres. La Houlière lui a donc adressé une nouvelle lettre, envoyée à l'adresse parisienne du marquis, faubourg Saint-Honoré. Par cette lettre du 5 octobre 1776, en plus de la précédente, nous apprenons que les nouvelles prétentions de William Wilkinson s'élèveraient à 330000 livres en dix ans, et que M. de Sartine allait lui écrire en faisant de nouvelles propositions, pour essayer de rabattre quelque chose sur cette somme.

Le 14 décembre 1776, La Houlière était à Nantes et adressa à nouveau un courrier au marquis de Langeron, pour annoncer la prochaine coulée de quatre canons, le premier le surlendemain, les trois autres dans la semaine suivante. Mais encore fallait-il par la suite les forer, ce qui ne pouvait pas encore se faire à Nantes. Et on voit réapparaître la rivalité entre de La Houlière et de Secval :

« Vous aurez été instruit que M. de Secval n'avait pas envoyé ici de foreur pour éviter la dépense qu'il aurait fallu faire à la Machine du Sr. Frerot, et M. de Secval a ordre de faire passer les canons faits ici à Ruelle pour y être forés; il eût cependant été moins coûteux de les forer à Rochefort, où la Marine a une forerie; on aurait évité le transport en remontant la Charente

48. Ibid. 
et leur renvoi à Rochefort, et l'épreuve y eut été faite authentiquement et comme la vôtre de Brest ${ }^{49}$."

Dans la même lettre, La Houlière faisait également une courte allusion à l'arrivée du Docteur Franklin, qui, ayant débarqué comme il est connu à Auray, semble donc être passé par Nantes avant de se rendre à Paris.

Le 16 décembre 1776 a été coulé le premier canon dans le four à réverbère du Sieur Frérot à Saint-Sébastien près de Nantes. La Houlière en rendit compte à Langeron le lendemain. Un second canon fut coulé le 19, puis un troisième le 24 décembre, le tout avec succès, et La Houlière était enfin satisfait de voir reconnue "sa méthode ". Suivit une quatrième coulée, et un procès-verbal en bonne et due forme, non daté, mais qui a été écrit par de Secval le 29 décembre 1776, dont voici le début :

"Nous Fougeroux de Secval, Chevalier de l'ordre royal et militaire de Saint Louis, lieutenant des vaisseaux du Roi et Officier d'artillerie de la Marine, de concert avec Monsieur d'Angenoust, Chevalier de l'ordre royal et militaire de Saint Louis, Chef de Brigade au corps Royal de l'Artillerie, en présence et aidé par Monsieur de Lahoulière, Chevalier de l'ordre royal et militaire de Saint Louis, Brigadier des Armées du Roi, et de Monsieur Boyard, ancien commissaire général de la Marine,

Conformément aux ordres à nous donnés par Monsieur de Sartine, Ministre et secrétaire d'Etat au département de la guerre, de reconnaître la méthode la plus certaine et la plus économique pour refondre les vieilles fontes, et les convertir en munitions de guerre, nous avons procédé comme il suit :

nous nous sommes servis du fourneau de réverbère que le sieur Frerot a établi à la côte Saint Sébastien près de Nantes. Il nous a paru à peu près le même que ceux dont se servent les anglais pour couler leurs canons en fonte de fer de seconde fusion; nous avons seulement estimé, d'après les plans de ceux-ci que nous a communiqués $M$. de Lahoulière, que celui du sieur Frerot pouvait en différer par une moindre intensité de chaleur, et qu'on remédierait aisément à cet inconvénient s'il était question d'en construire un nouveau $[\ldots]^{50}$."

Comme on peut le constater à la lecture des titres des participants et signataires du procès-verbal des essais de coulée, tant la marine que l'armée étaient représentées lors des essais. Mais il est question, dans la première partie du texte, de refondre des vieilles fontes pour les convertir en " munitions ", et non en canons. C'est, selon La Houlière, Monsieur de Secval qui aurait rédigé le procès-verbal des essais de coulée, dans un sens qui lui convenait mieux. Cet officier de l'artillerie de marine restait opposé à la nouvelle méthode de coulée des canons proposée par La Houlière. Il n'était pas le seul parmi les officiers de la marine royale, mais avait un orgueil particulier à propos de ses propres méthodes utilisées à Ruelle.

49. Médiathèque de Nantes, MS 506 C4. Lettres de M. de La Houlière au comte de Langeron sur la fonte des canons. Lettre du 14 décembre 1776.

50. Médiathèque de Nantes, MS 505 C3. Procès-verbal, décembre 1776. 
Dès le 29 décembre 1776, La Houlière faisait part de ses craintes au marquis de Langeron :

" Il est certain, Monsieur, que si M. de Secval en est cru ainsi que M. Potier il empêchera que l'on n'adopte cette manière de fondre, ou qu'il voudra s'en attribuer le mérite en seul : premièrement parce qu'il est persuadé que les canons de Ruelle, dont il croit avoir poussé la perfection au plus haut degré, sont aussi bons que les canons Anglois; secondement parce qu'il ne peut s'accoutumer à penser que les Anglois ayant, avant nous, trouvé une manière de faire des canons plus solides; troisièmement parce qu'il verrait avec peine un étranger travailler à Ruelle et exécuter ce que nous pouvons faire aujourd'hui sans lui, d'après ce que j'ai vu dans son établissement et les notions dont je lui suis redevable, et ce qui vient d'être fait; il est très certain qu'après avoir tâtonné et éprouvé différents mélanges et proportions de gueuses fondues à Ruelle et ailleurs avec des gueuses fondues avec des coaks en Angleterre ou en France, on fera des canons aussi bons que M. Wilkinson. Mais l'attachement aux anciens principes, l'habitude des Ouvriers ne fera-t-il pas des obstacles? Ne dira-t-on pas, si on ne fait pas aussi bien, que cette Méthode n'est pas aussi bonne; elle est cependant suivie par un gouvernement attentif à ce qui est intéressant pour la Marine et n'augmentera pas le prix actuel des canons ${ }^{51}$.

C'est à M. de Sartines à peser s'il vaut mieux faire opérer par M. de Secval ou par M. Wilkinson, s'il accepte les dernières propositions à lui faites.

M. de Secval rédige aujourd'hui [ 29 décembre 1776] le Détail de ces épreuves et cette rédaction fera naître des doutes ou fera voir une augmentation de Prix.

L'expérience des canons Anglois détruira les premiers, le Prix actuel des matières à Nantes et à Ruelle répondra à l'autre; j'hasarderai mes Observations, moi indigne, qui n'ai pas l'honneur d'être officier d'Artillerie de la Marine, ni de l'académie de Brest, ni frère de M. de Fougeroux, ni neveu de M. Duhamel.

Vous savez bien, Monsieur, qu'il y a plus de difficulté à faire agréer les meilleures choses qu'à les exécuter $[\ldots]^{52}$."

L'allusion à M. Duhamel concerne l'oncle de Fougeroux de Secval, qui était membre de l'académie de marine et inspecteur de celle-ci. Ici encore, on constate l'amertume que La Houlière manifestait lorsqu'il n'était pas en grâce auprès des puissants.

Vraisemblablement au début du mois de janvier 1777, La Houlière a écrit, comme annoncé dans la lettre que nous venons de citer, un document, qu'il n'a malheureusement pas daté, intitulé "Observations du Sieur de La Houlière sur les épreuves faites à Nantes ". Il y est à nouveau question de l'utilité de faire venir l'anglais Wilkinson en France pour y diriger un établissement de fonderie et de forage de canons. Faisant allusion aux établissements que le fondeur exploitait en Angleterre, il écrivait :

51. Souligné par La Houlière.

52. Médiathèque de Nantes, MS 506, C4. Lettres de M. de La Houlière au comte de Langeron sur la fonte des canons. Lettre du 29 décembre 1776. 
"C'est la connaissance du mélange convenable qui fait l'Art du Directeur de ces fonderies; ils le doivent à l'Expérience; des personnes aussi éclairées que MM. D'Angenoust et de Secval l'acquerraient en tâtonnant plusieurs Essais; ces MM. semblent aussi douter que la Marine angloise soit toute fournie en canons de $2^{\text {nde }}$ fusion.

Indépendamment de ce que j'ai vu et su par moi-même, $M$. le Duc de Guines s'est assuré, et a informé M. de Sartine et M. de Vergennes des fournitures immenses que $\mathrm{M}$. Wilkinson a fait et fait journellement pour la Marine angloise; en trois mois de temps, l'année dernière [en 1776], il est sorti de ses fonderies 2.388 pièces de tous calibres [...].

Je suis comme eux [MM. D'Angenoust et de Secval] très persuadé que cette méthode n'a été ni proposée ni discutée dans leurs Académies, les Artistes en ce pays ne les consultent guère; que M. Wilkinson, l'aîné, qui le premier a contracté avec l'Amirauté pour fournir des canons, l'a fait d'après ces expériences, et que les épreuves de ces canons, et leur bon service, leur a fait donner la préférence. $[\ldots]^{53}$."

Comme on le voit, la bataille que devait mener La Houlière pour faire aboutir son projet, qui, rappelons-le, consistait à faire venir l'anglais Wilkinson en France pour y construire et diriger une fonderie et forerie de canons selon les méthodes anglaises, n'était pas encore gagnée, et il s'en fallait de beaucoup. En plus, il devait sans arrêt manoeuvrer entre les différents acteurs que les uns et les autres mettaient en avant et protégeaient dans cette affaire. La Houlière comptait en particulier sur le marquis de Langeron, en qui il avait confiance, et auquel il ne cachait rien en tant que " camarade", pour ne pas divulguer certaines informations qu'il lui fournissait.

Ainsi il ressort d'une lettre que La Houlière a écrite le 9 janvier 1777 au marquis de Langeron que, décidément, les choses ne s'arrangeaient pas :

« Dans la conversation, Monsieur, que M. Dangenoust et moi nous eûmes hier soir [le 8 janvier 1777] avec M. de Sartines j'ai cru voir qu'il était disposé à faire suivre cette méthode de fondre par M. de Secval, je lui ai toujours parlé de la sûreté de l'opération, et de sa tranquillité personnelle avec M. Wilkinson, de la déclaration qu'il avait fait qu'il n'entendait avoir dans sa fonderie aucun inspecteur de Marine ou d'artillerie, et n'en connaissant d'autres que la Poudre pour ses canons.

Que n'étant pas convenable de déplacer M. de Secval de Ruelle, sa connaissance dans les fontes le rendant précieux à la Marine, le meilleur biais était de profiter des dispositions des principaux négociants de Nantes de se joindre à eux pour y établir une fonderie que $\mathrm{M}$. Wilkinson dirigerait en seul, et d'où on enverrait des ouvriers dans trois ou quatre mois, pour Ruelle, et qu'alors il aurait les moyens de fournir à tous les besoins de la Marine Royale et marchande, et même des canons pour le commerce que d'ailleurs, si les dernières propositions à M. Wilkinson avaient été acceptées, il ne pouvait décemment reculer et se dédire s'il arrivait; il ne voulut jamais me dire s'il lui avait écrit; il nous dit qu'il allait examiner le procès verbal [des essais de décembre] et je pense qu'il se propose d'en faire rapport au

53. Médiathèque de Nantes, MS 505, C3. Mémoire sur l'artillerie de Marine. Observations du Sieur de La Houlière sur les épreuves faites à Nantes, non daté. 
Conseil et de faire délibérer, attendu qu'il est question de dépenser 40.000 [livres] pour M. Wilkinson; que nos besoins et notre disette nous force à la lésinerie; néanmoins je lui ferai passer demain à Versailles, si je n'y vais pas moi-même, un plan qui lui remettra tout sous les yeux, d'après lequel il pourra et le Conseil se décider, et quand j'aurai tout dit, je n'aurai plus rien à dire $[\ldots]^{54}$."

Était-ce encore une manœuvre, et de qui et dans quel but? La Houlière voulait-il éloigner Monsieur de Secval de ses projets, en le déclarant précieux et indispensable à Ruelle? Voulait-il éviter un conflit potentiel entre de Secval et Wilkinson (qui ne manquera pas de se produire plus tard à Indret)? Ou bien revenait-t-il à son idée de faire réaliser la fonderie et forerie de canons aux environs de Nantes par « le commerce, en association avec Wilkinson "? Ce " commerce " est évidemment celui du Sieur Frérot, dont on a vu qu'il avait au moins une installation modeste permettant de couler et même de forer des canons à proximité de Nantes. Et on se souvient que dès les premières conversations entre La Houlière et John Wilkinson en 1775 en Angleterre, le second ne voulait pas traiter avec un gouvernement supposé éphémère, mais avec une entreprise privée. Le texte cité plus haut semble marquer un retour au point de départ, nonobstant le fait qu'il faudrait bien payer une forte somme à Wilkinson pour qu'il se déplace. Et ce n'est sans doute pas le " commerce " du sieur Frérot qui aurait été en état de faire cette dépense.

Le 11 janvier 1777, de La Houlière adressait une nouvelle lettre au marquis de Langeron :

"J'allai hier, Monsieur, à Versailles et remis à M. de Sartines mes réflexions sur la conversation que nous avions eu chez lui en présence de M. Dangenoust. J'en joins la copie pour que vous puissiez lui dire que je vous ai fait part de cette idée. Après lui en avoir fait lecture, je lui proposai de moi-même de les communiquer à M. de Secval que j'avais vu dans son antichambre et qui ne s'y trouva plus. J'aurais mieux aimé que cela lui fût communiqué en ma présence parce que mon Papier n'aura pu faire les répliques que j'aurais fait.

M. de Serval veut se réserver la satisfaction de peser en seul, se donner le mérite de faire épargner à M. de Sartines ce qui a été offert au Sr. Wilkinson, et les propositions d'épargne plaisent à tous les Ministres.

M. de Sartines me dit qu'il allait faire ses réflexions sur le Procès verbal d'épreuves. Je pense qu'il en parlera au Conseil; sur la proposition que je lui fis d'une lettre à écrire à MM. Les juges et consuls de Nantes, il me dit qu'il croyait plus convenable qu'il lui écrivissent; qu'au surplus je pourrais le voir mercredi de bonne heure, qu'il me dirait ses intentions.

J'avais vu M. le Cte de Vergennes qui incline toujours ainsi que vous et moi pour faire venir M. Wilkinson et qui parlera sur ce ton à M. de Sartines au Conseil. Il me fut impossible de voir M. le Cte de St. Germain.

Je vous cherchai partout sans vous rencontrer; je présume que vous irez aujourd'hui ou demain à Versailles.

54. Médiathèque de Nantes, MS 506, C4. Lettres de M. de La Houlière au comte de Langeron sur la fonte des canons. Lettre du 9 janvier 1777. 
J'aurai l'honneur de vous renouveler à votre retour mon fidèle et respectueux attachement ${ }^{55}$."

La rivalité entre La Houlière et Secval est des plus lisibles dans cette lettre. Le premier espérait encore que le comte de Saint-Germain plaiderait sa cause au conseil du roi. Quant au ministre Sartine, il semblait bien pratiquer une forme de non-décision ${ }^{56}$, au bénéfice de son officier de marine, Fougeroux de Secval.

\section{Vers la décision de construire la fonderie et forerie d'Indret}

Le brigadier-général La Houlière aurait-il été tenu à l'écart des dernières discussions et négociations qui ont précédé la décision de faire venir en France William Wilkinson pour contribuer à la création et participer à la direction de ce qui sera la fonderie et forerie de canons d'Indret? Le silence des copies de sa correspondance entre 11 janvier et le 18 mars 1777 peut le laisser penser. Il est possible également qu'il ait préféré se taire à l'égard de son " camarade ", le marquis de Langeron, et ne pas faire échouer, par trop d'interventions, les ultimes tractations menées par le ministre de Sartines avec Wilkinson. Toujours est-il que c'est ce ministre qui annonce, le 10 mars 1777, à son commissaire de la marine à Nantes, de la Villehélio, l'accord avec Wilkinson et l'arrivée prochaine dans cette ville de cet " habile fondeur":

"Je vous préviens, Monsieur, que le sieur Wilkinson, habile fondeur anglais, avec lequel il a été passé un marché dont je vous enverrai incessamment une expédition, s'est engagé à former dans les environs de Nantes, un établissement pour faire couler des canons et des mortiers de seconde fusion, et à procurer à nos ouvriers les connaissances nécessaires pour travailler avec succès d'après ses principes $[\ldots]^{57}$."

Ce n'est en réalité que le 11 mars 1777 qu'un traité fut signé par le ministre de Sartine, qui faisait de William Wilkinson le régisseur de la fonderie et forerie de canons à construire à Indret, avec le traitement annuel principal de 12000 livres françaises, soit 600 livres anglaises. Il paraît plus que probable que Wilkinson était présent à Paris ou Versailles pour apposer sa signature sur ce traité. Ce dernier est considéré comme provisoire par certains auteurs, qui lui préfèrent celui, plus définitif, signé le 17 mai 1777 entre les mêmes partenaires et aux mêmes conditions.

Les tractations ont donc fini par aboutir, et le 18 mars 1777, La Houlière en a fait part au marquis de Langeron :

55. Médiathèque de Nantes, MS 506, C4. Lettres de M. de La Houlière au comte de Langeron sur la fonte des canons. Lettre du 11 janvier 1777.

56. NAEGEL, Paul, Face à la non-décision : que faire?, Paris, Éditions Hommes et Techniques, 1987, $128 \mathrm{p}$.

57. Médiathèque de Nantes, 73593, C325. Lettres de la cour sur l'établissement de la fonderie d'Indret 1777-1789. Lettre du 10 mars 1777. 
«M. le Marquis de Langeron apprendra avec plaisir que l'arrangement entre M. de Sartines et M. Wilkinson a eu son entier accomplissement [...]. Le Ministre a pris le parti de faire exécuter aux dépens du Roy ce que le commerce était près de faire à Nantes pour son compte.

M. Wilkinson partira pour aller reconnaître et choisir son emplacement après demain jeudi [donc le 20 mars 1777]; il y a ordre d'acheter un terrain et un moulin pour la forerie $[\ldots]^{58}$."

La lettre de La Houlière du 18 mars 1777 prouve bien qu'à la date du 20 mars 1777, le terrain pour la future fonderie et forerie de canons n'était pas encore acheté. Il le sera dans l'île d'Indret qui, selon une lettre du ministre Sartine du 16 mai $1777^{59}$, "réunit tous les avantages qu'on peut désirer pour l'établissement de la fonderie à réverbère ${ }^{60}$ ".

William Wilkinson s'installa à Indret le 9 juin 1777, avec son interprète. La suite s'agissant de la construction de cette première fonderie et forerie a été largement décrite par plusieurs auteurs, dont Stéphane Girandier et Guy Gruais que nous avons cités.

Rappelons simplement que les travaux à Indret ont connu des lenteurs dues entre autres à des mésententes entre les acteurs sur le terrain. La mise au point de la forerie hydraulique, notamment, a posé problème par manque d'eau, et il aura fallu installer provisoirement un manège à chevaux pour mouvoir les forêts.

Puis vint la déclaration de guerre faite par la France à l'Angleterre en février 1778, et les risques qu'elle faisait peser sur la présence de William Wilkinson à Indret. Une dernière fois, La Houlière intervenait, le 29 mars 1778, auprès du marquis de Langeron, devenu général en 1762 :

« Mon voyage à Nantes, mon Général, est renvoyé par la même circonstance et les mêmes ménagements pour la personne qui ferait sans doute échouer l'entreprise si le Sieur Wilkinson pouvait repartir sans avoir monté son établissement, et toutes les circonstances paraissent seconder ses vues.

Au moment que j'ai reçu la déclaration qui a été faite à la Cour d'Angleterre, que j'ai supposé devoir opérer une rupture, j'ai représenté à M. de Sartines que ma présence pouvait être utile auprès de M. Wilkinson pour détruire dans son esprit la crainte des reproches que le Ministère britannique pourrait lui faire, pour animer son zèle et son activité, et pour lui suggérer tous les moyens de mettre au plus tôt l'établissement en état de fondre et de forer. Il m'a répondu qu'il n'était pas encore temps.

J'allai hier à Versailles pour lui représenter que si la frégate chargée de rapporter les moules de Dunkerque à Nantes était attaquée et prise dans la Manche, le sieur Wilkinson serait encore longtemps oisif; que le temps de son traité expirerait sans aucun fruit, et s'il ne montait et ne perfectionnerait

58. Médiathèque de Nantes, MS 506, C4. Lettres de M. de La Houlière au comte de Langeron sur la fonte des canons. Lettre du 18 mars 1777.

59. LA NicollèrRe-TEYIÈRE, S. de, Lettres de la Cour sur l'établissement de la fonderie d'Indret, Vannes, Imprimerie Lapolye, 1897, 33 p.

60. Ibidem, p. 6-7. 
pas cet établissement dans le temps pour lequel il s'est engagé de donner ses soins, on perdra tout ce qu'il en coûte. Je lui ai en conséquence suggéré d'écrire au Commandant et Commissaire de la marine à Dunkerque que si la frégate qui devait charges ces moules n'avait pas mis à la voile, ils aient à les faire partir au moment par voitures de terre pour Paris, d'où ils seraient envoyés en remontant la Seine au Canal d'Orléans, d'où ils descendraient par la Loire à Nantes, car il faut réparer à force d'argent le temps perdu.

J'ai lieu de croire que cette lettre aura été écrite aujourd'hui, et je souhaite qu'elle arrive à temps. Il m'a encore redit hier qu'il m'écrirait quatre ou cinq jours avant de me faire partir, d'où je conclus que M. de Secval est encore à Nantes, qu'il attend qu'il en soit parti pour m'y envoyer $[\ldots]^{61}$. "

Ce n'est finalement que le 15 août 1778 que de la Villhélio, commissaire de la marine à Nantes, a pu annoncer au ministre Sartine qu'il revenait d'Indret, et qu'il y avait vu couler et forer un canon de 6 (permettant de tirer un boulet de 6 livres), et qu'il était satisfait des opérations. Le ministre répondit le 28 août 1778 de la manière suivante : "Par votre lettre du 15, vous me marquez que vous avez vu couler et forer un canon du calibre de 6 , et que vous êtes satisfait de ces deux opérations. J'ai été bien aise de l'apprendre et de savoir que le moulin pourra commencer à travailler au moins d'octobre prochain $[\ldots]^{62}$."

Entre la visite du brigadier général de La Houlière, en juillet-août 1775 aux Wilkinson, en Angleterre, et la première coulée suivie du forage d'un canon, le 15 août 1778 , il se sera donc, à peu de chose près, écoulé trois années. C'est à la fois beaucoup et peu. L'établissement d'Indret dans sa première version était déjà assez considérable et supposait la maîtrise de plusieurs techniques (génie civil, hydraulique, fonderie à la houille, forerie) et leur mise en œuvre de manière coordonnée. Cependant, il est clair que, d'une part des tensions, voire des " manœuvres ", en rapport avec des considérations politiques, et d'autre part des relations inter-personnelles par moment conflictuelles entre les acteurs concernés, ont fait perdre du temps. À tout bien peser, cela n'aura peut-être retardé la mise en service de la fonderie et forerie d'Indret que de quelques mois.

Nous avons voulu montrer dans cette étude, sans reprendre ce qui a déjà été publié à propos de l'édification proprement dite de la fonderie et forerie de canons d'Indret, le rôle très important joué, bien avant toute construction effective, par le brigadier-général Marchand de La Houlière.

Pour les responsables de la marine royale, prendre en considération, les propositions, même éclairées et solidement étayées, d'un général de l'armée de terre, était sans doute très difficile. Mais ce qui caractérisait le brigadier-général Marchand de La Houlière était à tout le moins une certaine opiniâtreté, pouvant aller jusqu'à l'entêtement.

61. Médiathèque de Nantes, MS 506-C4, Lettres de Mr. de La Houlière au Comte de Langeron sur la fonte des canons, lettre du 29 mars 1778.

62. La NiCOlliÈre-TeYiÈRe, S. de, Lettres de la Cour..., op. cit., p. 26-27 
Les interventions de celui-ci ont été très nombreuses entre 1775 et le premier semestre de 1778, en premier lieu pour convaincre William Wilkinson de venir en France, et d'y apporter son savoir faire de fondeur et d'aléseur de canons, puis pour faire procéder à des essais de coulée comparatifs de canons, dans un four à réverbère existant dans les environs de Nantes, afin de prouver que les méthodes anglaises permettaient de réaliser de meilleures armes, et à moindre coût, que ceux fabriqués selon les techniques françaises traditionnelles, notamment à Ruelle. Ayant des " entrées à la Cour ", il est finalement arrivé, par l'intermédiaire du comte de Saint-Germain, ministre de la guerre, à convaincre le ministre Sartine, en charge de la Royale, du bien fondé de ses idées.

\section{RÉSUMÉ}

La création de la forerie de canons d'Indret, près de Nantes, a fait l'objet d'un certain nombre de publications françaises, dont notamment celles de Stéphane Girandier et Guy Gruais. Mais à une allusion près chez Girandier, le rôle du brigadier-général Mathieu Henri Marchand de La Houlière a été pour l'essentiel passé sous silence. Sans son acharnement, dès 1775, et ses nombreuses initiatives tant auprès de personnalités proches de la cour que d'officiers de la marine et de l'armée, la fonderie-forerie d'Indret n'aurait peut-être pas été créée.

Nous proposons dans ce travail de faire un récit des efforts faits par ce général d'infanterie, à partir notamment de copies jusqu'ici inexploitées de ses lettres, expédiées entre 1775 et $\mathbf{1 7 7 8}$, ainsi que d'écrits, peu connus en France, de l'historien anglais William-Henry Chaloner.

\section{ABSTRACT}

The creation of the gun drilling factory of Indret, in the surroundings of Nantes, gave way to several French research papers, amongst other the ones of Stéphane Girandier and Guy Gruais. But apart from an allusion by Girandier, the role of the Brigadier General Mathieu Henri Marchand de La Houlière, as from 1775, has been mainly eluded. However, the gun melting and drilling factory of Indret would not have been created without his commitment and numerous initiatives to monarchy and high ranking army and navy officers.

We propose in this study to write the story of the efforts deployed by this infantry general, on the basis of unexploited letters that he sent between 1775 and 1778, but also using papers, quite unknown in France, from the English history writer William Henry Chaloner. 